\title{
Hábitos de estudio y rendimiento académico: caso estudiantes de octavo grado 2017
}

Marta Isabel Delgado Mora*

Ubaldo García Lamus**

Artículo de reflexión

Fecha de Recepción: 12 diciembre 2017.

Fecha de Aprobación: 8 mayo 2018.

\section{Resumen}

El presente artículo pretende demostrar dequé manera pueden influir los hábitos de estudio en el bajo o alto rendimiento académico de los estudiantes. Además, el documento muestra los propósitos que se buscan al implementar algunas estrategias que coadyuven en la adquisición de hábitos de estudio para elevar el rendimiento académico de los estudiantes. El objetivo principal es identificar la incidencia que tienen los hábitos de estudio en el rendimiento académico de los estudiantes de grado octavo, en la asignatura Ciencias Naturales y Educación Ambiental, de 1a "Escuela Normal Superior Antonia Santos", ENSAS, de Puente Nacional, Santander.

Al respecto, se desarrolló una metodología de investigación acción con un enfoque mixto, el cual se basó en el estudio de categorías analizadas en el desarrollo de 6 guías relacionadas con: condiciones ambientales, planificación del estudio, comprensión lectora, velocidad lectora y memoria. Este trabajo posibilitó establecer la importancia de desarrollar unos buenos hábitos de estudio,
*Escuela Normal superior Antonia Santos - Puente Nacional - Santander Colombia

marthaisabel42@hotmail. com

$*$ Escuela Normal superior Antonia Santos - Puente Nacional - Santander Colombia profeubaldogarcia@gmail. com

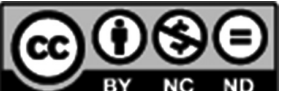


por cuanto estos influyen notable $\mathrm{y}$ favorablemente en el desempeño de los estudiantes. Se comprobó que los hábitos de estudio están íntimamente relacionados con un buen desempeño escolar, contribuyen a la disminución del fracaso escolar y ayudan en la vida personal de los estudiantes.

Palabras clave: Hábitos de estudio, rendimiento académico, planeación, comprensión de lectora, velocidad lectora y memoria. 


\section{Introducción}

En la gran mayoría de instituciones educativas en Colombia, según las pruebas externas, se refleja un alto índice de fracaso académico, el cual puede ser atribuible a la falta de hábitos de estudio en el ámbito escolar.

Se puede afirmar que estudiar es una labor, en muchas oportunidades un lujo y en otras un arte. La actividad intelectual de estudiar, debe estar acompañada con destrezas y habilidades que se adquieren con la práctica; la cual se precisa con una buena organización y adquisición de conocimientos en el proceso de enseñanza aprendizaje.

Este trabajo investigativo surge del compromiso profesional y social de las áreas de Ciencias Naturales y Matemáticas de la "Escuela Normal Superior Antonia Santos", de Puente Nacional. Los resultados obtenidos en las pruebas saber y los resultados de la autoevaluación institucional en el año 2015 y 2016, se constituyen en los referentes que permiten colegir que la falta de hábitos de estudio en los estudiantes de octavo grado, está estrechamente correlacionada con su rendimiento académico. Razón por la cual, la presente investigación buscó determinar la incidencia de los hábitos estudio en el rendimiento académico de los estudiantes de octavo grado de la mencionada Institución, en el tercer período académico del año 2017.

Así fue que, la relación entre hábitos de estudio y rendimiento académico se presenta de manera descriptiva e interpretativa, buscando un análisis con argumentos tanto empíricos como conceptuales. Además, el tema que se desarrolló en la investigación determinó que existe una relación directa entre los hábitos de estudio y el rendimiento académico, por lo que se constituye en un estudio fundamental para todas las Instituciones Educativas del país, y es un punto de referencia para aquellas que quieran implementar un plan de mejoramiento institucional, ya que la calidad educativa depende de una evaluación constante de los procesos pedagógicos que propicie la integralidad en el proyecto educativo y el ejercicio de la autonomía en la comunidad académica.

El trabajo centra la atención en la manera en que los alumnos estudian. Considerando que el tiempo que le dedican a estudiar, la organización, el sitio donde lo llevan a cabo, etc., conforman sus hábitos de estudio. Algunos estudiantes practican hábitos errados, que en vez de mejorar su nivel de aprendizaje les hacen retroceder. Por tanto, es importante tener buenos hábitos de estudio, y así propiciar que el alumno sea capaz de alcanzar un mejor y mayor aprendizaje.

La investigación se realizó con un grupo de 20 estudiantes del grado octavo de la Institución, del nivel de básica secundaria.

El problema planteado tiene que ver con ¿Qué incidencia tienen los hábitos de estudio en el rendimiento académico de los estudiantes de grado octavo en la asignatura de Ciencias Naturales, de la Escuela Normal Superior Antonia Santos de Puente Nacional?
En la gran mayoría de instituciones educativas en Colombia, según las pruebas externas, se refleja un alto índice de fracaso académico, el cual puede ser atribuible a la falta de hábitos de estudio en el ámbito escolar. 


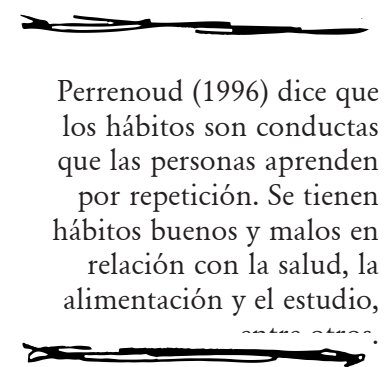

El objetivo principal fue identificar la incidencia que tienen los hábitos de estudio en el rendimiento académico de los estudiantes de grado octavo en la asignatura Ciencias Naturales y Educación Ambiental, de la referida Institución.

Para este fin, se llevó a cabo la aplicación de 6 guías categorizadas de la siguiente manera: Diagnóstico, Condiciones Ambientales, Planificación del Estudio, Comprensión, Velocidad Lectora y Memoria; dentro de las cuales se desarrollaron unas actividades concretas, que permitieron identificar que los estudiantes no tienen buenos hábitos de estudio $\mathrm{y}$, por ende, lo que se ve reflejado en su rendimiento académico y vida escolar.

\section{Fundamentación Teórica}

Éste trabajo investigativo, tiene su base teórica fundamental en algunos trabajos realizados sobre el particular, como los que se describen a continuación, donde se resalta el hecho de que los hábitos de estudio y rendimiento académico fue su principal objeto de estudio. Estos trabajos fueron determinantes a la hora de realizar un primer acercamiento al tema de investigación, así como para clasificar y clarificar las ideas e iniciar el camino investigativo.

Perrenoud (1996) dice que los hábitos son conductas que las personas aprenden por repetición. Se tienen hábitos buenos y malos en relación con la salud, la alimentación y el estudio, entre otros. Los buenos hábitos, sin duda, ayudan a los individuos a conseguir sus metas siempre y cuando estos sean trabajados en forma adecuada durante las diferentes etapas de la vida. El hábito, en sentido etimológico, es la manera usual de ser y, por extensión, el conjunto de disposiciones estables que, al confluir la naturaleza y la cultura, garantizan una cierta constancia de nuestras reacciones y conductas. Entonces, hábito es el conjunto de las costumbres y maneras de percibir, sentir, juzgar, decidir y pensar.

El hábito es un tipo de conducta adquirida por repetición o aprendizaje y convertida en un automatismo. El hábito, como la memoria y los instintos, es una forma de conservación del pasado.

Las dos fases del hábito son: 1) de formación y 2) de estabilidad. La primera, corresponde al periodo en que se está adquiriendo el hábito y la segunda, cuando ya se ha conseguido y se realizan los actos de forma habitual con la máxima facilidad y de manera automática (Velázquez, 1961).

Covey (2009) menciona que los hábitos son factores poderosos en la vida de las personas. Dado que se trata de pautas consistentes, a menudo inconscientes, de modo constante y cotidiano, expresan el carácter y generan nuestra efectividad o inefectividad. El hábito requiere de tres elementos para ser puesto en acción: a) el conocimiento, b) las capacidades y c) el deseo. Covey menciona que un estudiante no puede usar habilidades de estudio eficaces, hasta que no está teniendo buenos hábitos; y argumenta que, un individuo aprende con mayor rapidez y profundidad que otras personas debido 
a sus acertados hábitos de estudio. Además, reitera que el estudiar de forma eficaz y eficiente, consiste, más que en la memorización de hechos, en saber dónde y cómo obtener la información importante y la capacidad de hacer uso inteligente de la misma. Al igual que cualquier otra actividad, la habilidad y la dedicación son los puntos claves para el aprendizaje.

Por otra parte, Argentina (2013), en la tesis sobre "Relación entre hábitos de estudio y rendimiento académico en estudiantes de primero básico" (estudio realizado en el "Instituto Básico de Educación por Cooperativa", San Francisco La Unión, Quetzaltenango), demostró objetivamente que la práctica de buenos hábitos de estudio influye en el rendimiento académico,; es decir, que de los buenos hábitos de estudio depende el éxito que se obtenga durante el recorrido estudiantil hasta la vida profesional.

Enríquez Villota (2013) desarrolló una investigación en la ciudad de San Juan de Pasto, Nariño, titulada "Hábitos y técnicas de estudio en la Universidad Mariana”, donde concluyó que los estudiantes que adquieren hábitos de estudio y practican adecuadamente las técnicas en sus labores académicas, estarán en la capacidad de responder eficientemente a las diversas exigencias académicas.

García, Gutiérrez \& Condemarín (2005) señalan que la repetición constante de una misma acción, realizada bajo condiciones ambientales de espacio, tiempo y características iguales, favorece la acción de estudiar o la fuerza de voluntad para producir una conducta mecánica: el hábito. De tanto repetir, esta conducta se constituye en un comportamiento que modifica la actitud.

Una vez determinadas las categorías y subcategorías de análisis y examinando los principales referentes teóricos, nuestro grupo de investigación se inclinó por Francisco Fernández Pozar, quién ha realizado inumerables investigaciones, en cuanto a nuestro principal objeto de estudio: los hábitos de estudio y el rendimiento académico.

Fernández (2014) menciona como factores generadores de buenos hábitos de estudio, los siguientes:

\section{Condiciones Ambientales del estudio:}

a. Condiciones

Ambientales

Personales. Los condicionantes ejercidos por el ambiente que nos rodea (familia y compañeros) y por nosotros mismos (creencias, metas, etc.). Es, pues, necesario guiar al estudiante para que se defina y tome posiciones, con los tres círculos que se mencionan a continuación:

Ambiente Familiar. Las personas que conviven con el alumno ejercen un continuo influjo sobre él. Este influjo, en cuanto al rendimiento escolar se refiere, puede ser directo cuando sus familiares le ayudan o lo desaniman al hablarle del estudio; o indirecto, cuando la tónica general de armonía o desarmonía reina en casa del alumno.
Enríquez Villota (2013) desarrolló una investigación en la ciudad de San Juan de Pasto, Nariño, titulada "Hábitos y técnicas de estudio en la Universidad Mariana" 
Ambiente escolar. Compañeros y profesores constituyen el círculo vital del alumno durante la mayor parte del día. Los compañeros se pueden clasificar en expectativospasivos, activo- perturbadores y activo-constructivos. En cuanto a los profesores, cuando dada la situación actual de la relación educadoreducando y la idiosincrasia de la nueva generación, será interesante tener en cuenta tres notas:

- Ser verdadera "guía” para el estudiante. El concepto de profesorguía, conlleva a que los alumnos sepan que "pueden contar con él", acudir a él, considerarle como amigo.

- Hacer ver al estudiante la parte positiva, atractiva y simpática que tienen todas las asignaturas.

- Proporcionarle suficientes estímulos motores capaces de motivarlo a estudiar.

$\checkmark$ Ambiente personal. En este aspecto hay varios puntos que el estudiante debe tener muy claros.

- Llegar al convencimiento de que el estudio es una auténtica profesión, importante y digna, individual $\mathrm{y}$ socialmente.

- Hacerle entender que, con el estudio, el hombre se hace más capaz, más útil para algo y para alguien. Transmitirles esta idea a los alumnos es fácil, cuando el profesor mismo así lo cree.

- Que se convenza de que el trabajo de estudiar tiene dificultades. b. Condiciones ambientales físicas. El estado físico del cuerpo y el lugar donde se trabaja tiene mucho que ver con el rendimiento. De ahí que, sean importantes los siguientes apuntes:

- El cansancio, la falta de sueño, el hambre, el dolor de cabeza, de los ojos, etc., son serios obstáculos para un estudio provechoso. Por lo que, para estudiar, se necesita utilizar aquellas horas en las que el cuerpo esté más descansado.

- Estudiar en un lugar que permita la concentración.

- Tener mesa y silla de trabajo adecuadas, luz proveniente de la izquierda, temperatura aproximada a los $20^{\circ} \mathrm{C}$, buena ventilación, etc.

- Se debe lograr que el lugar de estudio sea algo personal, que genere comodidad y verdadero aprecio, un laboratorio donde el estudiante realice su trabajo personal.

c. Comportamiento académico. El estudiante, como profesional que es, también debe conocer y cumplir las normas de su empresa: "el colegio".

- Estar atento durante las explicaciones de los profesores.

- Preguntar al profesor cuando no comprende algo.

- Colaborar activamente en las tareas del colegio.

- Tomar apuntes durante las explicaciones del profesor. 


\section{Planificación del estudio}

a. Horarios. Los puntos fundamentales que giran alrededor del aprovechamiento del tiempo de estudio son:

- Confeccionar un horario de estudio personal.

- Incluir en ese horario todas las asignaturas

- Incluir en el horario periodos de descanso

- Cumplir verdaderamente el horario hasta que se convierta en un hábito.

b. Organización. Se deben tener en consideración los siguientes aspectos:

- Preparar todo lo necesario antes de sentarse a estudiar.

- Tener cada cosa en su sitio, da una positiva sensación de orden y seguridad.

- Estar satisfecho con la propia organización

\section{Utilización de materiales}

a. Lectura. La lectura es uno de los instrumentos principales de trabajo. Muchos de los problemas de rendimiento escolar tienen origen en una lectura defectuosa del alumno que no lee a una velocidad adecuada o que no comprende bien lo leído. Unas normas muy sencillas a este aspecto pueden ser:

- Leer por frases y no palabra a palabra.
- Leer con intención de mejorar la velocidad

- Al leer, saber distinguir los aspectos más importantes.

- Releer cuando algo no se ha comprendido bien del todo.

- Hacer pausas en la lectura para pensar y reflexionar sobre aspectos problemáticos.

b. Libros y otros materiales. El empleo de libros de texto y otros materiales de consulta, de ampliación, de apuntes, de gráficos, etc., debe tenerse en cuenta a la hora de la formación integral de los alumnos.

c. Esquemas y resúmenes. En el momento actual, en que cada día son más numerosos los contenidos culturales, en donde impera la velocidad; en que todo o casi todo se ofrece por fascículos, por dosis condensadas, es imprescindible que los estudiantes estén preparados para hacer síntesis. Los aspectos más sobresalientes en este apartado son:

- Subrayar en libros y apuntes los temas, palabras y puntos más importantes.

- Elaborar esquemas de toda la lección estudiada.

- Redactar un resumen de la lección, previamente esquematizada.

\section{Asimilación de contenidos}

Memorización. Muchos casos de alumnos de rendimiento escaso 
son atribuidos a una debilidad de memorización. Para superar esta deficiencia, ellos deben tener unas ideas muy claras respecto al acto de memorizar, que pueden ser las siguientes:

- Los alumnos deben tener confianza en su propia memoria.

- Comprender, antes de memorizar.

- Emplear el mayor número de entradas sensoriales para memorizar.

- Un método de memorización con excelentes resultados abarca estos pasos ordenados:

Lectura rápida de la lección o pregunta que se quiere aprender.

$\checkmark$ Volver a leer y detenerse en aquellos puntos que se consideran más importantes.

$\checkmark$ Subrayado de los puntos importantes.

$\checkmark$ Esquema escrito de la lección o pregunta, siguiendo las pautas del subrayado.

$\checkmark$ Elaboración del resumen.

Repetición oral y escrita del esquema o resumen.

b. Personalización. Por personalización se entiende, el tratamiento que se debe dar a cada alumno como individuo particular con sus características, y no como un número indiferenciado perteneciente a una masa. Como forma concreta de actuación personalizadora está el doble sistema del trabajo en equipo $\mathrm{y}$ trabajo personal.

c. Trabajo en equipo. Las ventajas de esta forma de trabajo son: desarrollo de hábitos de sociabilidad, colaboración, contraste de pareceres, ayuda mutua, etc.

\section{Características del Rendimiento Académico.}

En cuanto a rendimiento académico, se puede concluir que hay un doble punto de vista, estático y dinámico, que encierra al sujeto de la educación como ser social. En general, el rendimiento académico es caracterizado del siguiente modo:

a. El rendimiento en su aspecto dinámico responde al proceso de aprendizaje, como tal está ligado a la capacidad y esfuerzo del alumno.

b. En su aspecto estático comprende al producto del aprendizaje generado por el estudiante y expresa una conducta de aprovechamiento.

C. El rendimiento está ligado a medidas de calidad y a juicios de valoración.

d. El rendimiento es un medio y no un fin en sí mismo. El rendimiento está relacionado con propósitos de carácter ético que incluyen expectativas económicas, lo cual hace necesario un tipo de rendimiento en función del modelo social vigente. 


\section{a. Incidencia en el Rendimiento Académico.}

En nuestra sociedad, el éxito o el fracaso académico llevan consigo un sinnúmero de valoraciones de miedo. Ser "buen estudiante" es muchas veces tomado como sinónimo de "ser bueno", valioso, competente. En la comunidad educativa en general es una constante medir el valor del estudiante por los resultados académicos que obtenga. (Maslow, 1990, p. 50).

Para este trabajo investigativo la categoría de análisis "Rendimiento Académico" fue considerada como la correspondencia de la actividad escolar de los estudiantes en el área Ciencias Naturales con la (valoración) calificación obtenida, en concordancia con la escala valorativa nacional (Decreto 1290 de 2010) promulgada en Colombia por el Ministerio de Educación Nacional MEN. Escala que define como "Reprobatoria" la ubicación en el "Nivel Bajo" y como "Aprobatoria" la ubicación en los niveles "Básico, Alto y Superior"; así como la correspondencia con el sistema numérico de calificación del plantel descrito en el Sistema Institucional de Evaluación -SIE- (10 a 59èBajo; 60 a 79 èBásico; 80 a 89èAlto; 90 a 100è Superior) y, su equivalencia con la anterior escala nacional (Decreto 0230 de 2002), que definía como "Reprobatoria" la ubicación en los Niveles "Deficiente e Insuficiente" y, como "Aprobatoria" la ubicación en los Niveles Aceptable, Sobresaliente y Excelente. Es necesario aclarar que, las anteriores escalas valorativas están en estricto orden jerárquico, y van desde el nivel inferior hasta el nivel superior.

\section{PROPUESTA PEDAGÓGICA}

De acuerdo con la metodología de la investigación acción, se desarrollaron tres etapas fundamentales: la primera, tiene que ver con el diagnóstico; la segunda, con el diseño y la aplicación y, la tercera, con la evaluación.

\section{Primera Etapa: Diagnóstico.}

En esta fase se tuvo en cuenta el contenido del primer objetivo específico, que consiste en realizar un diagnóstico de los hábitos de estudio en los estudiantes de octavo grado en el primer periodo académico del año 2017 de la "Escuela Normal Superior Antonia Santos".

Para lograr este primer acercamiento con fines de diagnosticar el nivel en que se encuentran los estudiantes en sus hábitos de estudio, se propuso utilizar algunos instrumentos, de cara a la saturación de las categorías que enmarcan la investigación. En primera instancia, se planteó conocer los aspectos vivenciales de los estudiantes por medio de una encuesta que estuvo acompañada de una guía por desarrollar con los estudiantes, la cual pretende identificar en los estudiantes objeto de este estudio, aspectos relacionados con la visión que ellos tienen respecto a sus hábitos de estudio.

Para concluir esta etapa se propuso especificar, individualmente, los hábitos de estudio en los estudiantes, mediante un instrumento denominado 
Inventario de Hábitos de Estudio, de Fernández (2014). Este instrumento permite desarrollar una caracterización que es susceptible de análisis e interpretación amplia y mediante estos resultados, determinar los perfiles de hábitos de estudio presentes en los estudiantes objeto de la investigación.

\section{FASE 2. Diseño y aplicación}

Se dio paso a la segunda parte de esta investigación, que estuvo encaminada a diseñar y aplicar una propuesta que trata de impulsar aquellos hábitos que fueron identificados en la fase anterior. Para tal fin, se diseñaron guías en donde cada estudiante del grado octavo C de la Escuela Normal, desarrollo un trabajo personalizado; proporcionando a cada estudiante, los objetivos concretos por desarrollar, basados en el modelo de actuación que ofrece Fernández (2014),
"Aprender a Estudiar".

En el acápite de la fase 2: Diseño y aplicación, se desarrolló una propuesta basada en (6) talleres (Guías) que promovieron el uso adecuado de hábitos de estudio. Se presentó cada una de ellas, y allí, el estudiante realizó sus hábitos diarios en lo referente a condiciones ambientales, planificación del estudio, utilización de materiales y asimilación de contenidos.

Guía 2. Condiciones Ambientales Guía 3. Planificación del estudio Guía 4. Velocidad lectora Guía 5. Comprensión lectora Guía 6. Memoria

(Laguía número 1 fuela correspondiente al diagnóstico)

Dichas guías fueron evaluadas con la siguiente rúbrica, para dar validez a su desarrollo.

Tabla 1. Rúbrica de Evaluación

\begin{tabular}{|c|c|c|c|c|}
\hline NIVELES & CRITERIOS & $\begin{array}{l}\text { NIVEL } \\
\text { ALTO }\end{array}$ & $\begin{array}{l}\text { NIVEL } \\
\text { MEDIO }\end{array}$ & $\begin{array}{l}\text { NIVEL } \\
\text { BAJO } \\
\end{array}$ \\
\hline \multirow[b]{2}{*}{$\begin{array}{l}\text { CONDICIONES } \\
\text { AMBIENTALES }\end{array}$} & $\begin{array}{l}\text { Desarrolla hábitos de estudio adecuados conforme a la } \\
\text { dimensión y el elemento adecuado }\end{array}$ & & & \\
\hline & $\begin{array}{l}\text { Manifiesta la apropiación de condiciones ambientales } \\
\text { adecuadas para lograr un buen resultado en su rendimiento } \\
\text { académico }\end{array}$ & & & \\
\hline \multirow{2}{*}{$\begin{array}{l}\text { PLANIFICACIÓN DEL } \\
\text { ESTUDIO }\end{array}$} & $\begin{array}{l}\text { El tiempo que le dedica a las asignaturas es proporcionar a } \\
\text { su importancia }\end{array}$ & & & \\
\hline & Elabora su propio horario de estudio & & & \\
\hline \multirow{2}{*}{$\begin{array}{l}\text { UTILIZACIÓN DE } \\
\text { MATERIALES } \\
\text { (Velocidad y } \\
\text { Comprensión Lectora) }\end{array}$} & Sabe distinguir las partes importantes cuando lee & & & \\
\hline & Se preocupa por mejorar su calidad y velocidad de lectura & & & \\
\hline \multirow{2}{*}{$\begin{array}{l}\text { ASIMILACIÓN DE } \\
\text { CONTENIDOS } \\
\text { (Memoria) }\end{array}$} & $\begin{array}{l}\text { Procura poner en práctica y emplear lo que aprendió de } \\
\text { memoria para así aprenderlo mejor }\end{array}$ & & & \\
\hline & $\begin{array}{l}\text { Repasa a menudo lo que ha aprendido para así refrescarlo } \\
\text { y no olvídalo totalmente }\end{array}$ & & & \\
\hline
\end{tabular}

Fuente: Autores 
En esta apartado, se aplicó el tercer objetivo. Una vez culminada la aplicación de los instrumentos correspondientes a las dos primeras fases aplicados a la muestra objeto de estudio, se procedió a tabular los datos obtenidos de los estudiantes del grado octavo $\mathrm{C}$, respecto a su rendimiento académico, en el primer y segundo periodos académicos del año 2017, en el área de Ciencias naturales y Educación ambiental. La tabulación implicó el ordenamiento de esta información, la cual se clasificó atendiendo al desarrollo del tercer objetivo de la investigación que es evaluar el modelo de hábitos de estudio, a través de la comparación de los resultados académicos obtenidos por los estudiantes de octavo grado de la "Escuela Normal Superior Antonia Santos", en los periodos mencionados del año 2017.
Los datos se agruparon de acuerdo con los ítems establecidos en el sistema de evaluación institucional que tiene la ENSAS, "Escuela Normal Superior Antonia Santos", que son: SABER, SABER HACER y SER, lo que permitió y facilitó el análisis y la comparación de los resultados obtenidos por los estudiantes y, así, verificar con claridad la efectividad de la intervención que se aplicó a los estudiantes en cuanto a sus hábitos de estudio.

La comparación de estos datos demostró que, cambiar, mejorar e intervenir directamente en los hábitos de estudio en los estudiantes, se verá reflejado en su rendimiento académico.

Esta comparación se hizo mediante la presentación de cuadros individuales y gráficas, haciendo uso del software Microsoft Excel, como la siguiente tabla:

Tabla 2. Fase 3 Escala valoración institucional (Área Ciencias Naturales y Educación Ambiental)

\begin{tabular}{|l|c|c|c|c|c|c|c|}
\cline { 3 - 8 } \multicolumn{2}{c|}{} & \multicolumn{3}{c|}{ PRIMER PERIODO } & \multicolumn{2}{c|}{ SEGUNDO PERIODO } \\
\cline { 2 - 8 } \multicolumn{1}{c|}{} & ESCALA & SABER & HACER & SER & SABER & HACER & SER \\
\hline BAJO & $1,00-59,99$ & & & & & & \\
\hline BÁSICO & $60,00-79,99$ & & & & & & \\
\hline ALTO & $80,00-89,99$ & & & & & & \\
\hline SUPERIOR & $90,0-100,00$ & & & & & & \\
\hline
\end{tabular}

Fuente: Autores 
Para darle validez a la tabla anterior, se aplicó la prueba Chi cuadrado, que determinó la relación entre las dos variables estudiadas, hábitos de estudio y rendimiento académico.

\section{RECURSOS}

Productos. Los resultados que se pretenden conseguir con el desarrollo la presente propuesta son:

- Que los resultados de la investigación demuestren la existencia de una relación directa entre los hábitos de estudio y el rendimiento académico en el área de Ciencias Naturales y Educación Ambiental, de los estudiantes de octavo grado de la "Escuela Normal Superior Antonia Santos".

- Conocer las principales dificultades y posibles mejoras en las actuales falencias en los hábitos de estudio de los estudiantes de octavo grado de la ENSAS, las cuales se ven reflejadas en el nivel académico

- Proporcionar al docente y al estudiante líneas de acción relacionadas con la formación de hábitos, para la obtención de mejores resultados académicos

- Ofrecer información que oriente a cada uno de los involucrados en la toma de acciones de mejoramiento $\mathrm{y}$ toma de decisiones educativas

- Avalar una hipótesis, resultado de este estudio y planteada por los actores involucrados en esta propuesta

\section{METODOLOGÍA}

El tipo de investigación empleada para este proyecto es acción; define la investigación acción, pues es una reflexión sobre las acciones humanas y las situaciones sociales vividas por el profesorado, y tiene como objeto ampliar la comprensión (diagnóstico) de los docentes de sus problemas prácticos. Es decir, que las acciones van encaminadas a modificar la situación inicial con el propósito de lograr una comprensión profunda de los problemas. Según Murillo (2010), en la investigación acción otro de los grandes representantes, es Kemmis (1984) para quien el autorreflexión es una forma de indagación participativa del profesorado, el alumnado y la dirección en las soluciones educativas, con el objeto de mejorar la racionalidad de las propias prácticas de la comprensión de las mismas y las instituciones donde se realiza.

La presente investigación se enmarca dentro de un enfoque mixto, entendido como un conjunto de procesos sistemáticos, empíricos y críticos de investigación que implican la recolección y el análisis de datos cuantitativos y cualitativos, así como su integración y discusión conjunta, para realizar inferencias, producto de toda la información obtenida y lograr un mayor entendimiento del fenómeno bajo estudio. El método mixto combina al menos un componente cuantitativo y uno cualitativo en un mismo estudio o proyecto de investigación. En un sentido amplio se visualiza a la investigación mixta, como un continuo 
en donde se mezclan los enfoques cualitativo y cuantitativo, centrándose más en uno de estos o dándoles igual importancia, permitiendo utilizar las fortalezas de ambos tipos de indagación, combinándolas y tratando de minimizar las debilidades potenciales presentes.

Para este caso, la parte cualitativa hace un profundo análisis de la incidencia que tienen los hábitos de estudio en el rendimiento académico de los estudiantes, especialmente en el área de Ciencias Naturales y Educación Ambiental. Se busca explicar las razones de los diferentes aspectos del comportamiento y de cómo estos hábitos influyen directamente en el factor académico. Para esto, se utilizan instrumentos detallados como el diario de campo y la observación directa del grupo objeto de investigación.

En cuanto la población y la muestra, este estudio se desarrolló en la "Escuela Normal Superior Antonia Santos", del municipio de Puente Nacional, en Santander, Colombia, con estudiantes de grado octavo. Esta es una institución educativa de carácter oficial, más el ciclo de formación de maestros, administrada por la congregación de las Hermanas Terciarias Capuchinas de la Sagrada Familia, confesionalmente Cristiana Católica, y autorizada, legalmente, por el Ministerio de Educación Nacional.

La ENSAS se encuentra organizada en cuatro gestiones: directiva, pedagógica, administrativa- financiera y de comunidad. Cuenta con una población estudiantil actual de 1.238 estudiantes, que provienen no solo del área rural y urbana del Municipio mencionado, sino también de municipios aledaños, como Barbosa, Moniquirá, San José de Pare, Suaita, Guepsa, Jesús María y Vélez.

Para el objeto de la presente investigación se tomó una muestra de 20 estudiantes del grado octavo B, conformado por 38 estudiantes, de los cuales hay 26 mujeres y 11 hombres, vinculados directamente con la enseñanza de la asignatura de Ciencias Naturales y Educación Ambiental, específicamente, en los hábitos de estudios relacionados con el rendimiento académico.

En cuanto los instrumentos empleados para la investigación, se tuvieron en cuenta:

- La encuesta: se utilizó con el fin de caracterizar la muestra y hallar algunas opiniones sobre el tipo de hábitos que emplean los estudiantes.

- Diario de campo: sirvió para observar los procesos de adquisición de hábitos empleados por los estudiantes, durante la realización del proyecto.

- Observación directa: se empleó con el fin de evidenciar las acciones utilizadas por los estudiantes durante el desarrollo de la investigación, y dar cuenta de las debilidades y fortalezas de las mismas

- Rúbrica de evaluación: permitió conocer criterios, comparaciones y resultados de la investigación.

- Propuesta pedagógica: se constituye en una evidencia del reconocimiento que hacen los estudiantes de los hábitos de estudio en su cotidianidad, 
en el aula de Ciencias Naturales y Educación Ambiental. Se evidencia con la implementación de guías de trabajo que intervienen directamente los hábitos de estudio; estas guías trabajaron cada una de las subcategorías que trabaja Fernández Pozar, una de ellas la categoría de análisis fue las Condiciones Ambientales, se trabajó mediante el siguiente modelo:

Modelo 1. Encabezado de la guía de condiciones ambientales

\section{GUÍA No 2 CONDICIONES AMBIENTALES}

FECHA: mayo 02 de 2017

RADO: Octavo C

Duración: 2 Horas

Título: MEJORAMIENTO DE LAS

CONDICIONES AMBIENTALES

EN EL ÁMBITO ACADÉMICO
Objetivo: Identificar y establecer un ambiente adecuado de trabajo para desarrollar las labores académicas

\section{Recursos:}

- Taller

\section{Competencias:}

- Adecua un sitio de trabajo acorde a las necesidades académicas

Actividad: Leer, analizar y desarrollar las preguntas diseñadas con la mayor sinceridad posible.

Justificación del Taller. Motivar a las competencias lectoras para incentivar a mejorar los hábitos y el gusto por la lectura a través de la narración oral y la apropiación de la lectura.

Estrategia Pedagógica:

\section{HÁ B I T O S}

\section{Lee detenidamente los siguientes apartados para desarrollar la actividad}

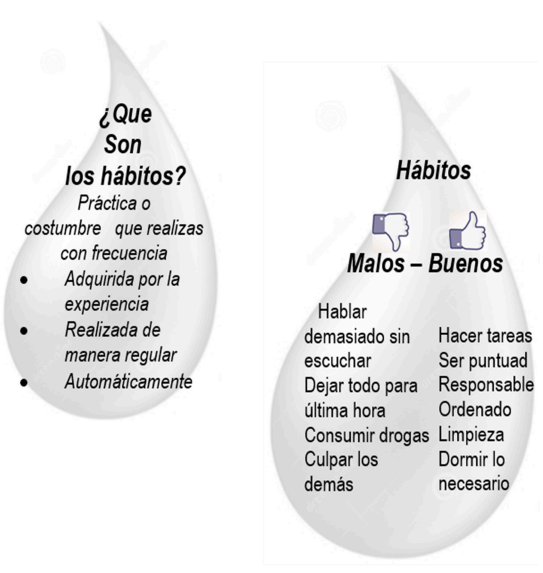

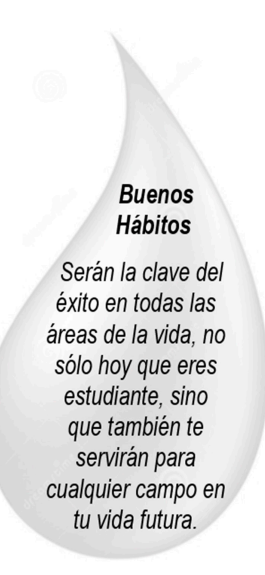




\section{RESULTADOS Y DISCUSIONES}

La aplicación de la primera guía de trabajo, el diagnóstico, planteó una situación: se trató de que los alumnos expresaran los logros o fracasos académicos individuales, por una parte, y los del colegio, por otra; es decir, se percibió como si los estudiantes tuviesen la conciencia de que ellos son parte activa de la Institución, de modo que sus logros los asumen como algo ajeno e independiente al ámbito del colegio.

En el desarrollo del primer objetivo de la investigación, se utilizó también el IHE de Pozar (2014), el cual determinó los perfiles individuales de los estudiantes en lo referente a las cuatro categorías principales: Condiciones Ambientales, Planificación del Estudio, Comprensión Lectora y Memoria.

Las Condiciones ambientales que se reflejan en el perfil de los estudiantes de octavo C de la ENSAS, evidenció que la gran mayoría se posicionaron en un nivel BAJO. De este modo, los estudiantes no procuraban tener un mejor espacio y actitud para su estudio; la Planificación de Estudio posicionó la gran mayoría de estudiantes en un nivel MEDIO, lo que significa que los estudiantes no organizaban su tiempo ni sus recursos de una manera óptima; la Comprensión Lectora estuvo en nivel BAJO, para la mayor parte de estudiantes, lo que significaba que no demostraban competencias claras y, en la categoría de Memoria, la mayoría de estudiantes se ubicaron en un nivel BAJO, lo que expresaba que los estudiantes tenían pocos elementos confiables a la hora de elaborar resúmenes y esquemas.

En ese contexto, se llevó a cabo un plan de intervención en cada uno de los estudiantes con el desarrollo de guías, estrategia que buscaba incidir, directamente, en cada una de las categorías determinadas en el diagnóstico. Objetivo que se efectuó con la orientación directa de los docentes investigadores, en el que los estudiantes, a medida que iban desarrollando cada una de las guías (como queda registrado en los diarios de campo), iban corrigiendo cada una de sus falencias, en cuanto a las categorías de Hábitos de Estudio y, por ende, esto se iba reflejando en su rendimiento académico.

Este rendimiento académico fue el último objeto de estudio de esta investigación, ya que se demostró que, este mejoró, sustancialmente, en el segundo periodo académico; tal hecho quedó evidenciado por medio de matrices aplicadas a cada una de las guías, y al seguimiento del rendimiento académico de los estudiantes en el primero y segundo periodos académicos, en el área de Ciencias Naturales, verificado con la posible asociación entre las variables categóricas Hábitos de Estudio y Rendimiento Académico mediante la prueba Chi Cuadrado (X2). Esta prueba se aplica para encontrar la relación entre variables cualitativas (categóricas), a través de la prueba de hipótesis.
La aplicación de la primera guía de trabajo, el diagnóstico, planteó una situación: se trató de que los alumnos expresaran los logros o fracasos académicos individuales, por una parte, y los del colegio, 
Una de las debilidades de este trabajo de investigación, fue el poco tiempo de aplicación de los instrumentos, ya que de haberse realizado en un periodo más largo se hubieran podido evidenciar más y mejores resultados; otra dificultad estuvo relacionada con la imposibilidad de controlar algunos aspectos de los hábitos de estudio como lo referente al trabajo desarrollado en su propia casa y el cumplimiento de horarios, aunque se dieron directrices claras en el desarrollo de las guías es casi improbable controlar estos aspectos individualmente.

Este trabajo investigativo, al igual que los tenidos en cuenta en el estado del arte del mismo, demostraron y llegaron con corta diferencia al mismo resultado, que los hábitos de estudio influyen directamente en el rendimiento académico de los estudiantes; aquí se hace un llamado de atención hacia las prácticas educativas desarrolladas en la ENSAS hasta el momento, ya se hace urgente hacer un análisis respecto a la formación que se orienta en torno a valores sociales como la corresponsabilidad, el sentido de pertenencia y la identidad institucional.

Con respecto al grupo seleccionado para este estudio, los estudiantes de octavo C de la ENSAS, se sugiere desarrollar un estudio en cada uno de los grados de la Básica Secundaria, Media Vocacional e, inclusive, el Ciclo Complementario, y se diseñe un instrumento similar que permita tener una noción de Hábitos de Estudio de cualquier estudiante del colegio, generando una regla general de interpretación que permita diagnosticar y proponer intervenciones de mejoramiento de los hábitos de estudio de los estudiantes.

Este trabajo de investigación marca la importancia del desarrollo de habilidades o hábitos de estudio adecuados para el buen desempeño del estudiante de la ENSAS. Asimismo, se propone en este estudio que los maestros alienten a los estudiantes a probar diferentes estrategias, desarrollar una variedad de herramientas útiles y descubrir qué es lo que mejor les funciona tanto para su aprendizaje, como para lograr las metas de su vida.

El proyecto educativo institucional PEI de la ENSAS y, más específicamente su modelo pedagógico conceptual constructivista, el cual se apoya en principios de la Pedagogía Amigoniana, demanda de los estudiantes un trabajo integral, ya sea individual o grupal, planeado y orientado por el docente. Aquí es donde los hábitos de estudio se convierten en facilitadores e integradores del proceso de enseñanzaaprendizaje; por cuanto, ellos son los encargados de impulsar los recursos cognitivos, físicos y ambientales que poseen los estudiantes; pues teniendo claro que cada persona estudia y aprende de diferente manera, resulta más oportuna la creación y organización de ambientes, en donde se privilegie la construcción y aprehensión de aprendizajes duraderos $\mathrm{y}$ trascendentales.

Además, este estudio permite demostrar que los estilos cognitivos personales de cada estudiante, impactan de una 
manera muy directa los procesos educativos de toda la Institución Educativa. Es por eso que estos, deberían pensarse en función de los sujetos que los viven y los desarrollan, es decir, los estudiantes; de allí deberían surgir las diferentes estrategias pedagógicas que, según el modelo pedagógico del colegio, desarrollen y promuevan una educación de calidad; donde a cada estudiante se le permita construir sus propios modelos de aprendizaje, basados en una toma de decisiones crítica y argumentada, incluyendo además las nuevas tecnologías de la información y comunicación, las cuales requieren otras habilidades por parte del estudiante y nuevos retos para el docente, tales como capacitarse más allá de su quehacer pedagógico y disciplinar para así llegar a incorporar nuevas competencias y estrategias en sus prácticas educativas llegando, inclusive, hasta a darle mayor autonomía al estudiante y de tal manera, estimular que participe y se involucre en auto formación, concluyendo de por sí en nuevos métodos de evaluación.

\section{CONCLUSIONES}

El principal objetivo de este proyecto fue identificar la incidencia que tienen los hábitos de estudio en el rendimiento académico de los estudiantes de grado octavo en la asignatura Ciencias Naturales y Educación Ambiental, de la Escuela Normal Superior Antonia Santos, de acuerdo con esto, durante el desarrollo y ejecución de este trabajo se demostraron ciertos aspectos que nos llevaron a establecer ciertas conclusiones.
En primera instancia, respecto a los Hábitos de Estudio, se pudo evidenciar que para los estudiantes son un aspecto primordial y determinante a la hora de obtener buenos y excelentes resultados académicos, además, por medio del desarrollo de la primera fase del proyecto se pudo derivar que un alto porcentaje de los estudiantes del grado $8^{\circ} \mathrm{C}$ no presentaban niveles satisfactorios a la hora de evaluar sus hábitos por medio de un instrumento que el principal referente teórico Fernández Pozar nos proponía.

En lo relativo al rendimiento académico que para el caso de la Escuela Normal Superior Antonia Santos esta categorizado por niveles de Bajo, Básico, Alto y Superior, niveles que se obtienen mediante la evaluación integral de los estudiantes en su Saber, Saber Hacer y Ser; se logró determinar que los niveles Bajo y Básico que presentaban 20 de los 38 estudiantes objeto de este estudio, estaban relacionados con los niveles de $\mathrm{Mal}$ y No Satisfactorio que estos mismos estudiantes presentaban a elaborar en ellos el perfil de hábitos de estudio.

A sí mismo, por medio de la implementación y desarrollo de guías específicas, direccionadas a cada uno de los niveles de hábitos que Fernández Pozar mediante su Inventario de Hábitos de Estudio clasificaba, se pudo demostrar que dichos hábitos se pueden reactivar o perfeccionar si se llevan a cabo acciones concretas en cada uno de los aspectos definidos, para llegar a obtener unos hábitos de estudios considerados como aceptables. 
Comparando los resultados obtenidos por los estudiantes en el primer periodo académico, periodo en el cuál se elaboró la primera fase de la investigación con los del segundo periodo del año 2017, periodo durante el cual se llevó a cabo la intervención directa en el grupo de estudiantes, se pudo evidenciar un progreso, en cuanto a que un número significativo de estudiantes mejoró su promedio final de calificaciones haciendo que disminuyeran los niveles de Bajo respecto a su rendimiento académico, originando entonces la principal conclusión de este proyecto que los hábitos de estudio inciden directamente en el rendimiento académico de los estudiantes del grado 8 C de la Escuela Normal Superior Antonia Santos de Puente Nacional Santander.

\section{Referencias}

Argentina, V. (2013). Relación entre bábitos de estudio y rendimiento académico en estudiantes de primero básico" (estudio realizado en el Instituto Básico de Educación por Cooperativa San Francisto La Unión, Quetzaltenango. Giuatemala.

Elliot, J. (1994). La investigación-acción en educación. Madrid: Morata.

ENSAS. (2016). Autoevaluación Institucional. PUente Nacional: ENSAS.

Fernández, F. (2014). Inventario de hábitos de estuio. Madrid, España: Tea.

García, C., Gutierrez, G., \& Condemarín, G. (2005). A estudiar se aprende: Metodología se estudio sesión por sesión. México: Alfaomega.

Kemmis, S., \& McTaggart, R. (1988). Cómo planificar la investigación acción. Barcelona: Laertes.

Krauskopf, D. (2000). Participacion Social y Desarrollo de la adolescencia. Costarica.

Mora, J. (1998). Acción tutorial y orientación educativa 5a Edición. Mexico: Narcea: S.A.

Murillo, J. (2010). Métodos de Investigación en Educación Especial. 3a. Educación Especial.

Negrete, J. A. (2007). Estrategias para el Aprendizaje. México: Noriega Editores: Limusa.

Padres, E. d. (2014). Los hábitos de estudio y su importancia en el éxito escolar. Catacaos : Escuela de padres. 